\title{
HEED Protocol using a Cluster based V2V Communication
}

\author{
M. Karpagam* \\ Department of Electronics and Communication Engineering, Sri Krishna College Of Engineering and Technology, \\ Coimbatore -641008, Tamil Nadu, India; karpagam@skcet.ac.in
}

\begin{abstract}
Objectives: Wireless sensor Networks employs a set a sensor nodes to monitor the parameters of the environment. The sensory information collected by the sensor nodes can be retrieved with the help of Mobile Nodes fixed upon vehicles. Existing scenarios engage both single-hop data transfer from Sensor nodes that lie within the mobile network range or profound participation of network margin nodes. These nodes are at the peril of quick energy collapse resulting in loss of network connectivity and decrease in network lifetime. The main idea behind this paper is to enhance the network lifetime and security. Methods/Statistical Analysis: The proposed HEED (Hybrid energy efficient Distributed Clustering) protocol reduces the overall network operating cost and energy disbursement associated with the multi hop data recovery process while also ensuring fair energy utilization among Sensor nodes and prolonged network lifetime. In multi hop network, the nodes closer to the sink node are involved in almost all data transmission, hence their energy would be quickly drained out. Since the energy depletion at sink node is more than other nodes, energy holes are created. Therefore other nodes in the VANET are disconnected from the network which reduces the network lifetime. To enhance network lifetime routing protocols are introduced in addition to HEED protocol which provides efficient clustering. To improve the security Intrusion-Tolerant Routing protocol (INSENS) protocol is used, which uses multipath technique in order to make the network resilient to attacks. Findings: The proposed HEED protocol elects the cluster head periodically based on a metric which is based on parameters like residual energy of the node, closeness of its neighbors and the degree of the node. The number of iterations involved in HEED protocol is very less and it achieves literally consistent distribution of cluster head allotment across the network involving a low level of information overhead. With suitable limits on node density, intra and inter cluster transmission ranges, this protocol can assure connectivity of clustered networks. Simulation results prove that this protocol is efficient in enhancing the lifetime of the network and sustaining scalable data aggregation. In case of Distributed clustering algorithms it is the responsibility of the base station to collect the information gathered by the cluster heads. In this work each and every sensor node broadcasts it accessibility to its surrounding. Based on the broadcast information the cluster head belonging to the specific cluster is identified in parallel. INSENS protocol is used to enhance network security. Simulation results prove that this protocol is efficient in enhancing the security of the network. Application/Improvements: This study proposes centralized, HEED_INSENS protocols to solve the coverage problem in VANETs. It is also useful for applications that require data aggregation and those where all data originated from sources should reach the sink without prior aggregation.
\end{abstract}

Keywords: Sensor Nodes, Network Overhead, Network Lifetime, HEED, INSENS

\section{Introduction}

Vehicular communication is the recent trend in Vehicular Adhoc Network where many new research works can be brought about. Data Exchange between vehicles in VANET is to facilitate route scheduling, highway safety and additional non-safety applications. A vehicle can communicate with another vehicle or to Roadside Unit. A cluster network could overcome these problems. Clustering is a technique to group nodes. Each cluster has the following nodes member node, cluster Head and cluster Gateway. Cluster head is principal node of a cluster

${ }^{*}$ Author for correspondence 
and it is accountable for coordinating all cluster members in the cluster. A Cluster gateway can be in touch with nodes belonging to different clusters. The major challenge for clustering algorithms is to maintain steadiness in a highly vibrant environment. The competent clustering algorithms should diminish the number of clusters forming and to maintain the current cluster structure and keep the overhead at the minimum level. For efficient clustering stable clustering is required. Here the main goal is to enhance cluster stability where vehicles change their speed and direction. In this work, a novel clustering approach is used with the aim of increasing the network stability and the network life time.

\section{Materials and Methods}

An innovative algorithm to create stable clusters to carry outrouting based on clustering approach and to improve the performance of VANETs. This routing based on clustering approach classifies the nodes based on the location and path information to form stable clusters and nominates cluster head based on an algorithm which depends on multiple metrics ${ }^{1}$. The chance of a node becoming a cluster head is determined by a value called suitability value $\mathrm{u}$, which is calculated based on information regarding mobility from its neighbors. The suitability value would be higher for a node if it has larger number of stable neighbors with the distance to their neighbors being minimum and have quicker speed to the average speed. Those nodes with higher suitability value have a higher prospect to be nominated as a cluster head. This method minimizes the overhead of re-clustering and guide to an efficient hierarchical network topology. A clustering mechanism for maintaining the connectivity in $\mathrm{VANET}^{2}$. An innovative algorithm based on clustering formaintaining the connectivity in vehicular ad-hoc networks is projected (AODV-CV). Here the maintenance of cluster connectivity is achieved by establishing stable and extended existing clusters as much as possible. The key initiative of cluster head nomination mechanism is based on the average speed of the vehicles moving in the same direction and the formation of virtual mobile cluster. Results prove that AODV-CV provides better maintenance of cluster connectivity and performances in the freeway where the vehicles' speed limit is within $120 \mathrm{~km} / \mathrm{h}$.

The dynamic change in topology effects the effective time of routing ${ }^{3}$. Hence routing in VANETs is complex.
During the route discovery process AODV broadcasts a route request message (RREQ). It generates many unused routes between a source and a destination node. To improve the performance, the VANET is made into small clusters with long Cluster Head duration. The AODV protocol is improved by exchanging broadcasting by RREQ packets with the forwarding of RREQ packets to Cluster Heads and there by managing routing by Cluster Heads and Gateway Nodes. This paper focus on improving the performance of AODV by enhancing the existing protocol by generating stable clusters and performing routing by Cluster Heads and Gateway nodes. A novel clustering algorithm based on weight to enhance the performance in the wireless networks ${ }^{4}$. In this algorithm the maximum jump from the cluster head to its member located farther apartis two jumps which will guarantee that other non cluster head node is ruled by only one cluster head which are its neighbors within two jumps. For the calculation of weight every node compares its own weight with that of its neighbors. Finally the cluster head would be the node which has the largest weight. In this approach speed is taken into account, in addition to the position and route to precisely identify nodes showing identical mobility prototype and group them into a cluster. A novel approach to nominate the Cluster Head $(\mathrm{CH})$ based on various metric was used. Suitability value is calculated using this technique. This ensures the e nominated cluster head to stay connected with its peers for the highest period of time. Hence if a node has larger number of stable neighbors with the distance to their neighbors being minimum and have quicker speed to the average speed then it would be having the more probability of being elected as a cluster head.

HEED is similar to the basic plan of LEACH where residual energy is the key parameter and network topology features (e.g. Degree of a node, distances from its neighbors) are only used as less important parameters to split tie among members and cluster heads ${ }^{5}$. A vehicle model based on cluster approach for vehicle contact, this paper pay attention on the development of clustering technique, by considering the vehicle density, speed and position of the vehicles to decrease the delay overhead ${ }^{6}$. The election of cluster head is dependent on parameters like speed and position of the vehicles. The switching technique employed for switching the cluster head is that whichever vehicle has more speed than cluster head is chosen as new cluster head. 


\section{Problem Definition}

In Vehicular Ad-hoc Network energy consumption and security has becomes a primary concern. In network involving multiple hops, the nodes close to the sink will be more congested as they are involved in transfer of data from the nodes located far away. Thus the power level of the nodes located closer to the sink node will be depleted very quickly when compared to nodes that are located far away from the sink node. This results in the creation of energy holes as the depletion of energy of the sensor nodes is non uniform. The consequence is that the other nodes get detached from the sink which impairs the VANET. So the main focus in VANET should be to prevent energy holes by balancing the energy expenditure of the sensor nodes. This leads to the depletion of network lifetime and cause network overhead. So to overcome these problems routing protocols are introduced.

\section{Methodology}

Network lifetime and network scalability of wireless sensor network can be enhanced by implementing topology control. An efficient topology control method is to cluster the sensor nodes. This paper proposes a new scattered clustering approach for lifetime extended ad-hoc sensor networks. The only assumption that is made in this work is accessibility of numerous power levels in the sensor nodes. No assumption is made regarding either the presence of infrastructure or the capabilities of the node.

The proposed work serves to enhance the lifetime of a network. The main idea behind enhancement of network lifetime is to distribute the consumption of energy by the cluster head. The algorithm which involves the election of cluster head is completed after programmed number of iterations. This work involves very minimal overhead information. HEED relies mainly on two metrics to elect the cluster head. The first metric is based on the residual energy remaining in each sensor node. Energy is lost in a sensor node while performing functions like sensing the necessary quantity, processing the sensed information and communicating the processed information. The energy remaining in the sensor node after performing the above said functions is referred to as residual energy. The second metric is inter and intra cluster communication. In this paper the first metric is used to elect first set of luster heads and the second metric is used to resolve contention between them. Contention refers to the situation where a node falls within the range of more than one cluster head.

Range refers to the power level at which transmission is done to make intra cluster broadcasting and to exchange information related to the clustering. This range at which communication is done is referred to as power level of the cluster. The power level of the cluster must be set to lowest power level of the sensor node so as to increase the spatial reclaim. The higher power levels must be preserved for inter-cluster communication. Those reserved higher power levels must be able to cover at least three times the diameter of a cluster. The power level of the cluster provides an indication of the number of clusters present in a wireless sensor network.

The cost of intra cluster communication is owing to the cluster size, uneven allowable power level for interring cluster communication cost. In all the other clustering protocols which deals with enhancement of energy efficiency every node can separately make a decision of its responsibility, so these algorithms does not ensure best set of elected cluster heads. The algorithm proposed in this paper plays a significant role in minimizing the energy consumed by the sensor nodes and therefore contributes to enhancing network lifetime.

Let Tclus denote the duration of the clustering process. Tclus actually specifies the time required to create a cluster by the clustering protocol. Let Tnet specify the operation time of the network. Thus a fresh cluster is formed every Tclus + Tnet seconds. The selection of cluster head is influenced by the radius of the cluster. When the cluster radius is small it would require larger number of cluster heads.

Let Nit represent the number of iterations required for completing the clustering process at each node. The time taken to complete each step be represented as tnew, this time duration should be sufficient enough to receive communication from any neighbor within the range of each cluster. Let Cint represent the opening percentage of cluster heads surrounded by $n$ nodes. The exact value of percentage of cluster heads cannot be predetermined. Cint only represents the opening percentage of cluster heads and is in no way has an direct influence on the final clusters. The first phae of this algorithm starts with each node calculating the probability of that particular node fetching the position of cluster head, CHprob.

$$
\mathrm{CHprob}=\mathrm{C}_{\text {int }} \times\left(\mathrm{E}_{\text {res }} / \mathrm{E}_{\text {maxi }}\right)
$$


Where $\mathrm{E}_{\text {res }}$ is the residual energy level estimated for a node, and $\mathrm{E}_{\text {maxi }}$ is the maximum energy which is normally alike for all nodes. The value of CHint should be chosen such that it should not be less than a threshold value $\mathrm{E}_{\text {min }}$, which is chosen to be inversely proportional to Emaxi. The node that has the $\mathrm{CHprob}$ as equal or greater than 1 will become the Cluster head for the cluster. The minimum power mandatory for a node is represented by PwrMin.. Let the average minimum reachability power (Amrp) which is the mean of the minimum power levels required by all $\mathrm{R}$ nodes within the cluster range to reach is

$$
A m r p=\frac{\sum_{n=1}^{R} P w r M i n}{R}
$$

Amrp gives a superior approximation of the cost involved in communication where each sensor node is permitted to choose the required power level to arrive at its cluster head. The Amrp of a node specifies the intra-cluster communication energy consumption if that particular node becomes a cluster head.

Every node is elected to become a cluster head with the probability CHprob. After the completion of the ith iteration the set of uncertain cluster heads, Sch, is set to \{cluster heads after step i - 1 [new heads selected in step i] of Sch is elected as the cluster head. The algorithm then proceeds to the next step.

The HEED algorithm consists of the following phases:

\subsection{Initialize}

- $\mathrm{Snbr}=\{\mathrm{v}: \mathrm{v}$ lies within my cluster range $\}$

- Compute and broadcast cost to $\varepsilon$ Snbr

- $\mathrm{CH}_{\text {prob }}=\max \left[\left(\mathrm{C}_{\text {prob }} \times \mathrm{E}_{\text {residual }} / \mathrm{E}_{\text {max }}\right), \mathrm{P}_{\text {min }}\right]$

- is.final. $\mathrm{CH}=$ false

\subsection{Main Processing}

Repeat

- If $\left(\left(\mathrm{S}_{\mathrm{CH}}=\{\mathrm{v}: \mathrm{v}\right.\right.$ is a cluster head $\left.\left.\}\right) \neq \Phi\right)$

- my_cluster_head = least.cost $(\mathrm{SCH})$

- If (my.cluster.head = NodeID)

- If $(\mathrm{CHprob}=1)$

- Cluster.head.msg (NodeID, final.CH.cost)

- is.final $\mathrm{CH}=$ True

- Else

- Cluster.head.msg (NodeID, tentative.CH.cost)

- ElseIf $(\mathrm{CHProb}=1)$
- Cluster.head.msg (NodeID, final.CH.cost)

- is.final. $\mathrm{CH}=$ True

- ElseIfRandom $(0,1) \leq \mathrm{CHprob}$

- Cluster.head.msg (NodeID, tentative.CH.cost)

- $\mathrm{CHprev}=\mathrm{CHprob}$

- $\mathrm{CHprob}=\min (\mathrm{CH}$ prob $\times 2,1)$

- UntillCHprev = 1

\subsection{Finalize}

- If(is.final. $\mathrm{CH}=$ False)

- If $\left(\left(\mathrm{S}_{\mathrm{CH}}=\{\mathrm{v}: \mathrm{v}\right.\right.$ is a Final head $\left.\left.\}\right) \neq \Phi\right)$

- my _ cluster_head = least.cost (SCH)

- join.cluster(cluste.head_ID, NodeID)

- Else cluster_head.msg(NodeID,finalCH.cost)

\section{INSENS Protocol}

INSENS is a protocol to prevent intrusion from a malicious node. INSENS meaning Intrusion Tolerant Routing protocol has two forms. The first one is the basic form and the second one is the enhanced version of the first one. The invasion of the outside node is blocked by Enhanced INSENSE protocol through confirmation of neighbor node using a global key, pair-wise key and cluster key.

Enhanced INSENS (INSENS) is operated using various steps which involves echo, exchange of key, route requests, and setup steps. Global key is injected by the INSENS protocol to each sensor node to obstruct an external invasion. An encrypted message is used by the echo step which employs the injected global key to block invasion from an external node. Using the global key an echo message is composed and broadcasted by every sensor node. When the neighbor sensor node receives the echo message it validates the received message with its own global key after which it produce pair wise key with the node sending the messages. The pair wise key is embedded into an echo back message and is then broadcasted.

$$
\begin{aligned}
& \mathrm{ECHO} \| \mathrm{E}_{\mathrm{GK}}\left(\mathrm{ID}_{\mathrm{x}} \| \text { nonce }\right) \\
& \text { ECHOBACK } \| \mathrm{E}_{\mathrm{GK}}\left(\mathrm{ID}_{\mathrm{y}} \| \text { nonce }+1 \| \mathrm{K}_{\mathrm{y}, \mathrm{x}}\right)
\end{aligned}
$$

Equation (3) represents the echo message and (4) is the echo-back message. ECHO and ECHOBACK are the message type, $E G K$ is encrypted using the global key, IDx and $I D y$ refers to the identity of nodes $\mathrm{x}$ and $\mathrm{y}$, nonce is a random number, and $K y, x$ is the Pair wise key shared 
between node $\mathrm{x}$ and node $\mathrm{y}$. Each node produces a cluster key after the echo step and forwards its own cluster key to each neighbor node using pair wise key of its own. After the completion of key exchange the base station produces an REQ message by using its own cluster key to setup a routing path setup. The format of the REQ message is as follows.

$$
\mathrm{REQ}\left\|\mathrm{ID}_{s}\right\| \mathrm{E}_{\mathrm{CK}_{s} \mathrm{~s}}\left(\mathrm{OHC} \| \mathrm{ID}_{\mathrm{BS}}\right)
$$

Here, (3) is the format of REQ message, IDs refers to the identity of the sender node, $C K \_s$ is the cluster key of the sender node, OHC represents the one-way hash chain (OHC) to prevent re-use and looping of the information, and IDBS is the identity of the Base Station, i.e., the boundary of the preferred routing path. The routing path arrangement process using the REQ message is shown in Figure 1.

Once the exchange of key is completed, the base station sends a REQ message to establish the routing path and the value of one way hash chain is incremented by 1 . After the key exchange step is completed, the BS broadcasts a REQ message in order to setup the routing path and it increments the OHC by 1 . When a node receives the REQ message, the authentication is done by its neighbors by employing the Cluster key of the sender node and their own one way hash chain (a). Once the nodes complete the verification step of REQ messages, it broadcast the encrypted REQ message using its own Cluster Key, and then it increments its own one way hash chain by 1 . Wireless Sensor Networks repeats this algorithm in order to set the routing path.

\section{Flowchart}

The proposed method identifies a false REQ message through the Base station and each node, which has interactive verification, and blocks it to prevent the intruder node. Base Stations produces each pair-wise key which is shared with each node for interactive verification before the network is configured. The REQ message checks to establish whether it is real or fake using a three-step authentication process. Figure 2 specifies the series of steps involved in the authentication process. The authentication steps are as follows.

- Verification of the Sender node verification

- Hold on for REQ messages from the Neighbor nodes

- Perform Interactive Verification

\section{Results}

The results are taken for network lifetime and security to verify the HEED performance is better than the existing method.

Figure 3 shows that the proposed HEED INSENS which has higher network lifetime than the Panel protocol and AODV. Figure 4 shows that the proposed HEED_INSENS has better security than the Panel protocol and AODV.

\section{Summary and Conclusion}

We have studied the problem of an energy and security in VANETs. First, we have characterized energy based on a

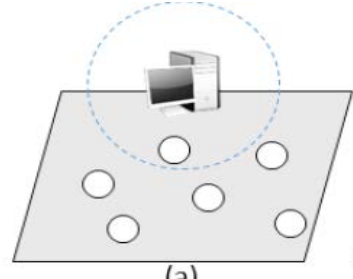

(a)

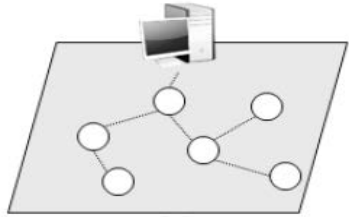

(c)

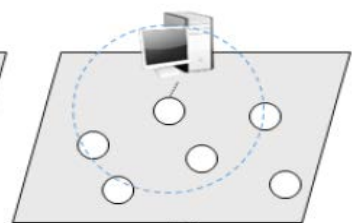

(b)

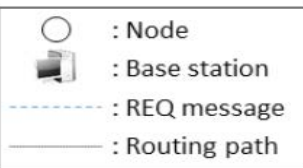

Routing path

Figure 1. Routing path setup process of INSENS. 


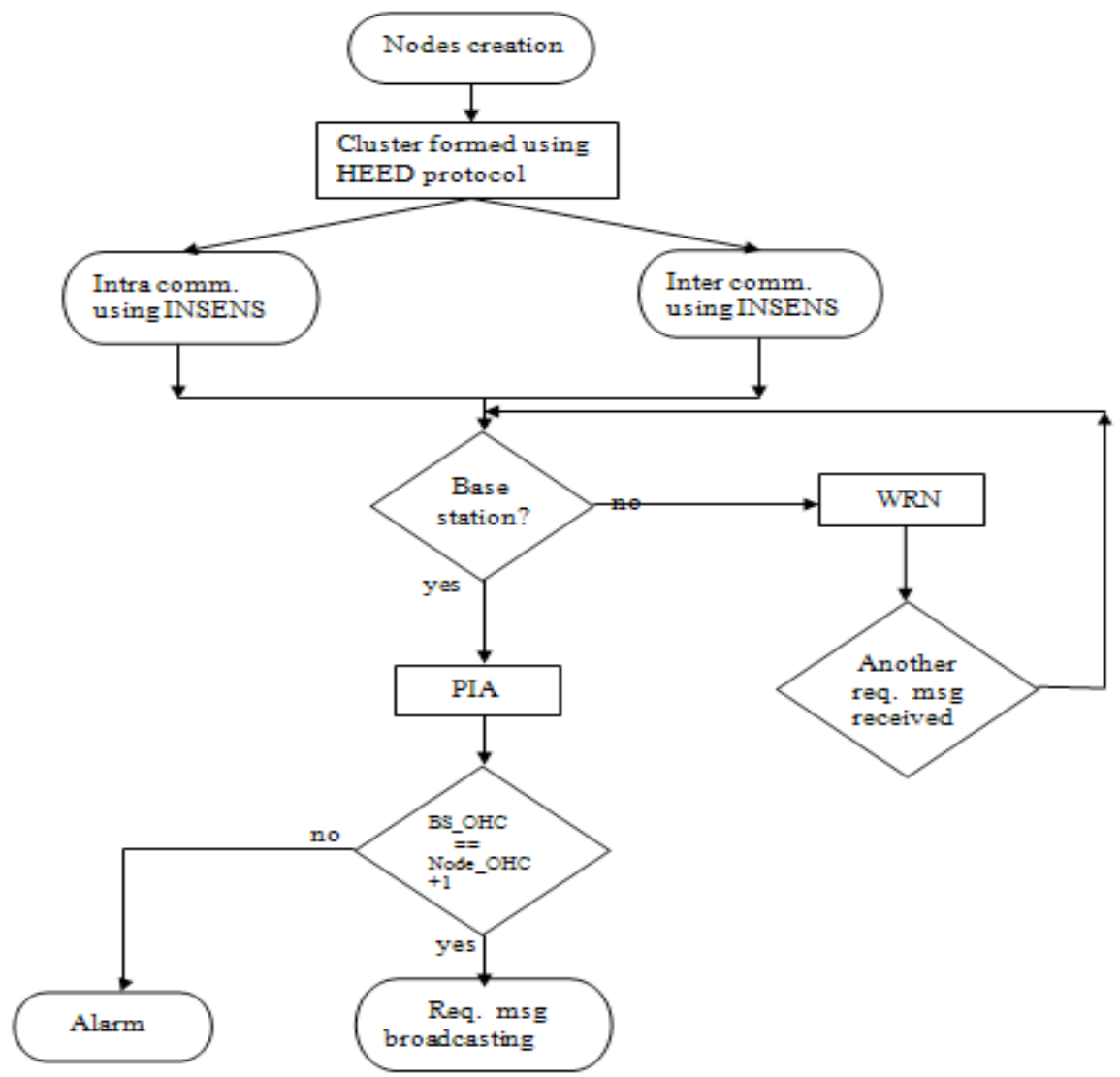

Figure 2. Data flow of simulation.

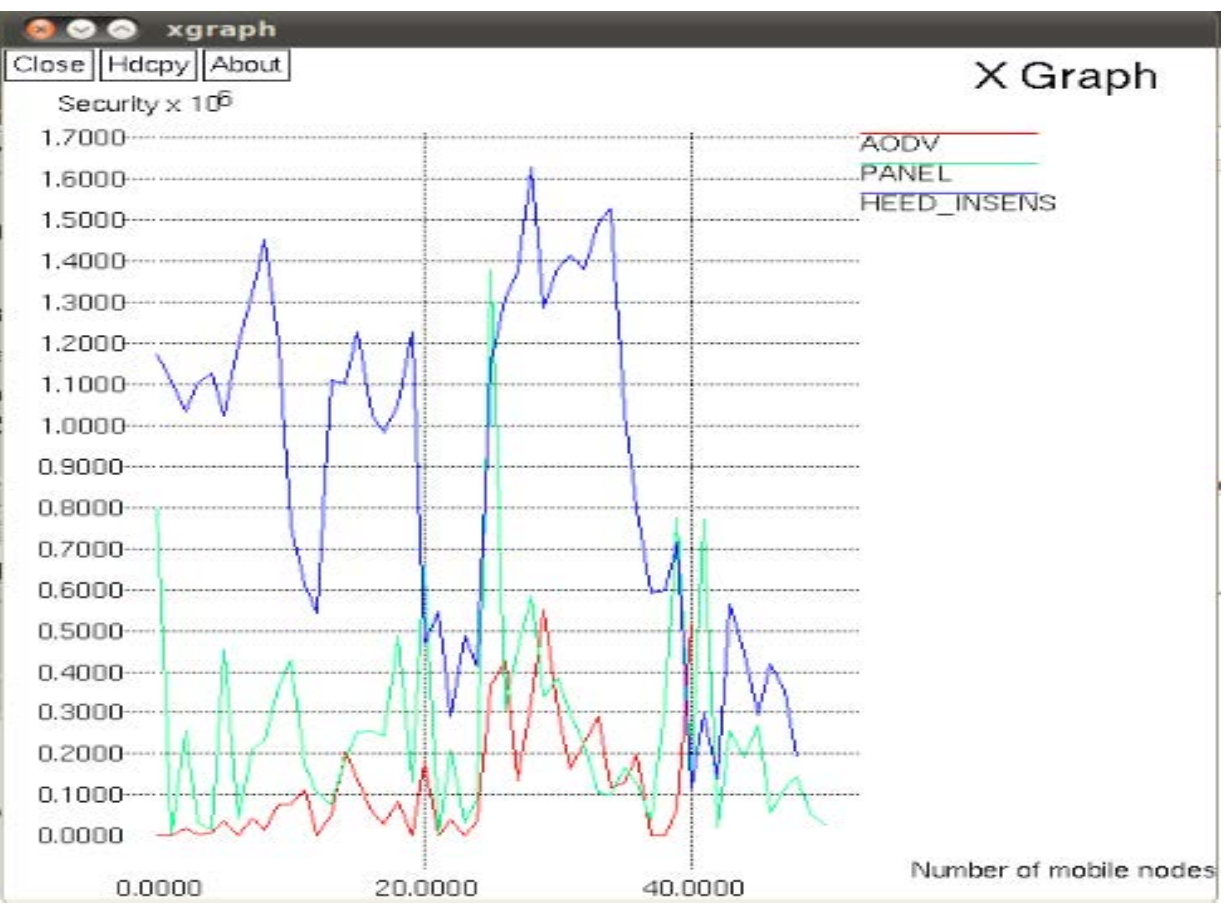

Figure 3. Network Lifetime graph. 


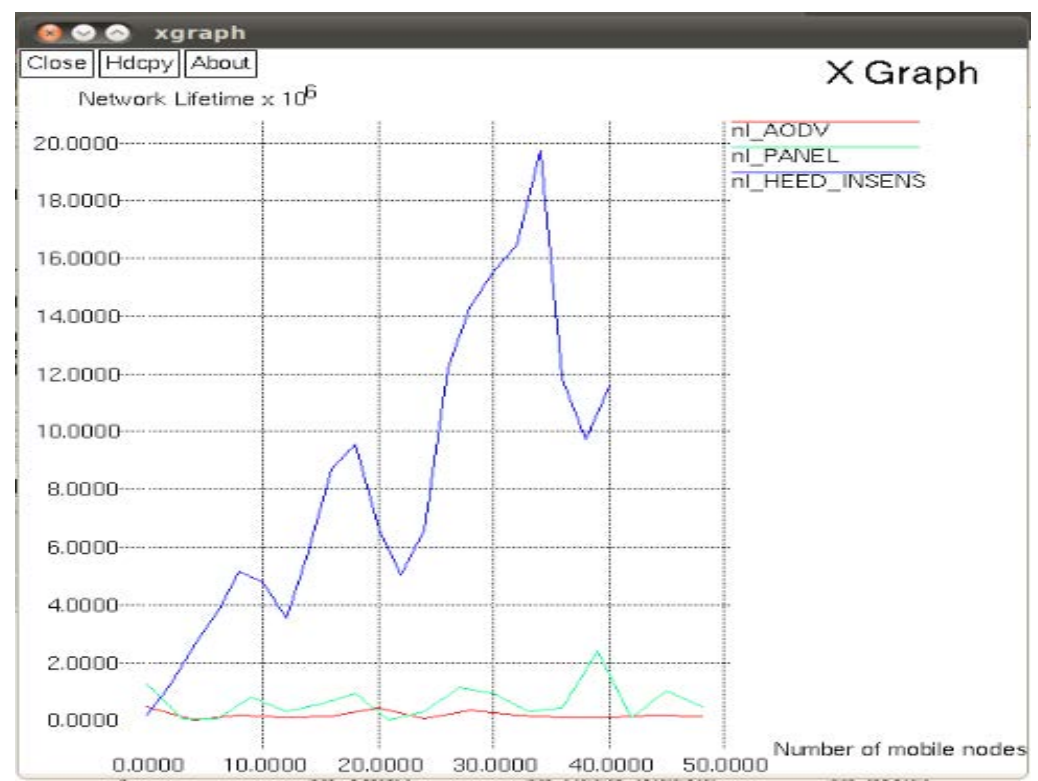

Figure 4. Security graph.

geometric analysis of the intersection of sensing disks of cluster groups. We have also computed a bound on the cluster field. Second, we have proved that coverage implies connectivity of the cluster members. Third, we have proposed centralized, HEED_INSENS protocols to solve the coverage problem in VANETs. It is also useful for applications that require data aggregation and those where all data originated from sources should reach the sink without prior aggregation. Our simulation results show that HEED_INSENS is more secured than EXISITING METHOD, with respect to the number of active sensors required for coverage and network operational lifetime.

\section{References}

1. Merentitis A, Kranitis N, Paschalis A, Gizopoulos D. Low Energy Online Self-Test of Embedded Processors in Dependable VANET Nodes. IEEE transactions on dependable and secure computing. 2012; 9(1):86-100. https://doi. org/10.1109/TDSC.2011.17

2. Konstantopoulos C, Pantziou G, Gavalas D, Mpitziopoulos A, Mamalis. A Rendezvous-Based Approach Enabling
Energy-Efficient Sensory Data Collection with Mobile Sinks. IEEE Transactions on Parallel and Distributed Systems. 2012; 23(5):809-17. https://doi.org/10.1109/ TPDS.2011.237

3. Zhang D, Li G, Zhen K, Zhao-Hua Pan XM. An EnergyBalanced Routing Method Based on Forward-Aware Factor for Wireless Sensor Networks. IEEE Transactions on Industrial Informatics. 2014; 10(1):766-73. https://doi. org/10.1109/TII.2013.2250910

4. Xing G, Wang T, Xie Z, Jia W. Rendezvous Planning in Wireless Sensor Networks with Mobile Elements. IEEE Transactions on Mobile Computing. 2008; 7(12):1430-43. https://doi.org/10.1109/TMC.2008.58

5. Besbes H, Smart G, Buranapanichki D, Kloukinas C, Andreopoulos Y. Analytic Conditions for Energy Neutrality in Uniformly-Formed Wireless Sensor Networks. IEEE Transactions on Wireless Communications. 2013; 12(10):4916-31. https://doi.org/10.1109/ TWC.2013.092013.121649

6. Liu J, Jiang X, Nishiyama H, Kato N. On the Delivery Probability of Two-Hop Relay MANETs with Erasure Coding). IEEE Transactions on Communications. 2013; 61(4):1314-26. https://doi.org/10.1109/ TCOMM.2013.020413.120198 